MUCOSAL IMMUNOLOGY

\section{TLRs get rhythm}

As well as detecting pathogenic organisms, Toll-like receptors (TLRs) coordinate homeostatic responses to commensal bacteria. Chambon and colleagues now report that TLR expression by intestinal epithelial cells (IECs) is under circadian control, leading to oscillatory immune signalling in response to the microbiota. Notably, in the absence of the microbiota the circadian clock is disrupted in IECs and mice develop metabolic disturbances.

To examine whether commensal bacteria are involved in circadian responses, the authors compared temporal gene expression profiles in IECs from control and microbiota-depleted mice. IECs from microbiota-depleted mice showed disrupted circadian control of several nuclear receptors, including PPARa (peroxisome proliferator-activated receptor- $\alpha$ ), REV-ERB $\alpha$ and ROR $\alpha$ (retinoic acid receptor-related orphan receptor- $\alpha$ ), and of components of the circadian clock machinery. Notably, depletion of the microbiota also led to systemic metabolic effects, with animals showing increased blood levels of glucose, triglycerides and free fatty acids, and decreased production of insulin.

Further analyses showed that these metabolic disturbances resulted from increased corticosterone synthesis by ileal IECs, which was caused by increased PPARa expression in these cells. Indeed, microbiotadepleted mice with an IEC-specific deletion of PPAR $a$ showed normal regulation of circadian clock components and did not show any metabolic disturbances. Microbial products promoted the activation of JUN N-terminal kinase (JNK) and the activator protein 1 (AP-1) transcription factor JUN in IECs, and JUN was shown to repress PPARa. However, although JNK and JUN showed a circadian mode of activation, their levels of gene and protein expression did not change over time.

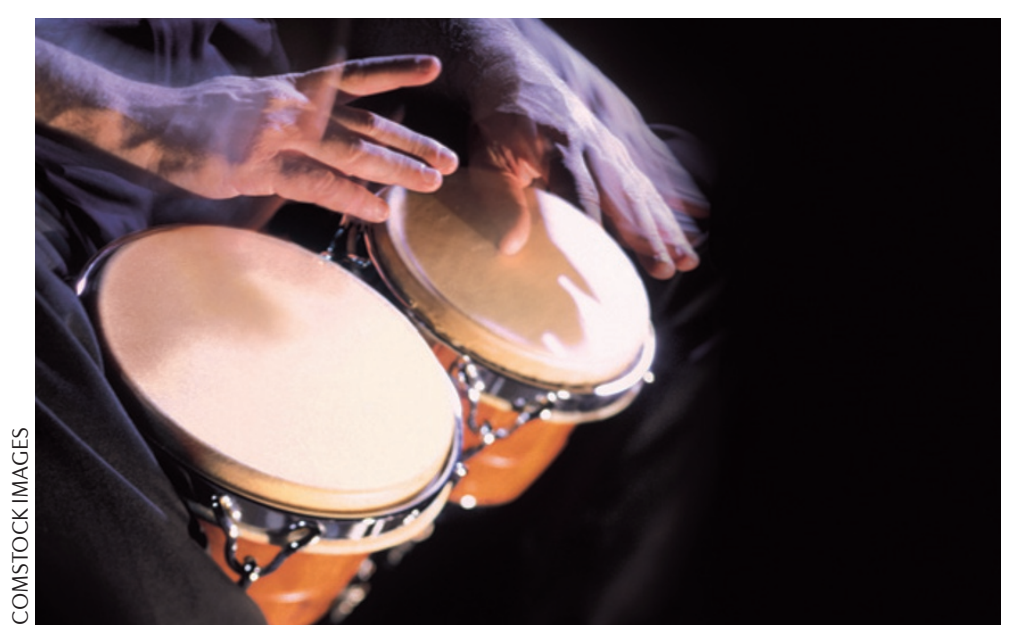

Instead, the authors found that the expression of TLR1 to TLR5 and also TLR9 (but not TLR6 and TLR7) is under circadian control in IECs. This explains how microbiotaderived products can activate JNK and JUN and repress PPAR $\alpha$ in a circadian manner. The temporal transcription of TLRs in IECs was shown to be activated and repressed by the alternate binding of RORa or REV-ERBa, respectively, to a conserved site in the TLR gene promoters. Using a bioinformatics approach, the authors found that a large number of genes $(>2,000)$ that are expressed by IECs contain the RORa and REV-ERBa DNA-binding site. This suggests that many of the genes that are involved in homeostatic IEC responses are controlled in a microbiota-dependent circadian manner. Indeed, the authors confirmed that several immune mediators that are important for maintaining epithelial barrier integrity are regulated in this way in IECs.

Taken together, the data show a key role for the microbiota and TLRs in controlling circadian responses in IECs. Such circadian control of IEC responses could be important for coordinating homeostatic IEC functions with behavioural activities in mice.

Yvonne Bordon

ORIGINAL RESEARCH PAPER Mukherji, A. et al. Homeostasis in intestinal epithelium is orchestrated by the circadian clock and microbiota cues transduced by TLRs. Cell 153 , 812-827 (2013)

FURTHER READING Sheiermann, C., Kunisaki, Y. \& Frenette, P. S. Circadian control of the immune system. Nature Rev. Immunol. 13, 190-198 (2013) 